EPJ Web of Conferences 41, 05028 (2013)

DOI: $10.1051 /$ epjconf/20134105028

(C) Owned by the authors, published by EDP Sciences, 2013

\title{
Femtosecond UV-pump mid-IR probe spectroscopy of the ultrafast photodissociation of azide radicals from an azido- iron(III) complex
}

\author{
Hendrik Vennekate ${ }^{1}$, Dirk Schwarzer ${ }^{1}$, Joel Torres-Alacan ${ }^{2}$, and Peter Vöhringer ${ }^{2}$ \\ ${ }^{1}$ Max-Planck-Institut for biophysical Chemistry, Am Fassberg 11, 37077 Göttingen, Germany \\ ${ }^{2}$ Institute for Physical and Theoretical Chemistry, University of Bonn, Wegelerstraße 12, 53115 \\ Bonn, Germany, p.voehringer@uni-bonn.de
}

\begin{abstract}
The ultrafast photolysis of the cation complex $\left[(\text { cyclam-ac }) \mathrm{FeN}_{3}\right]^{+}$is studied by femtosecond spectroscopy with ultraviolet excitation and mid-infrared probing. Immediately after UV absorption, the excited complex undergoes internal conversion and azide dissociation within 2 ps. The subsequent vibrational relaxation in the electronic ground state and geminate recombination of the fragments take place on time scales of 13 and $20 \mathrm{ps}$, respectively.
\end{abstract}

\section{Introduction}

Iron in exceptionally high oxidation states, i.e. $\mathrm{Fe}(\mathrm{IV}), \mathrm{Fe}(\mathrm{V})$, and $\mathrm{Fe}(\mathrm{VI})$, is implicated as transient intermediates in the catalytic cycles of many heme proteins like those responsible for the enzymatic oxidation in bio-organisms. Usually, the high oxidation state at the intermediate's iron center is difficult to detect, let alone to isolate it in significant amounts. Therefore, it is of paramount importance to develop synthetically accessible model systems containing highly oxidized Fe that are stable under laboratory conditions for a sufficiently long time to facilitate an in-depth study of their electronic and molecular properties including their spectroscopies. In this paper, we report on the femtochemistry of the compound, $\left[\left(\right.\right.$ cyclam-ac)Fe $\left.{ }^{\mathrm{III}} \mathrm{N}_{3}\right] \mathrm{PF}_{6}\left(\{\mathbf{1}\} \mathrm{PF}_{6}\right)$, where cyclam-ac represents the 1,4,8,11-tetraazacyclotetradecane-1-acetate ligand. The cation complex $\{\mathbf{1}\}$ might act as a photochemical precursor for the nitrido species, $\left[\left(\text { cyclam-ac) } \mathrm{Fe}^{\mathrm{V}} \mathrm{N}\right]^{+}\{\mathbf{3}\}\right.$, containing the metal at the oxidation state $+\mathrm{V}$. Hence, it constitutes an ideal low-molecular weight model system for heme biochemistry involving super-oxidized iron. [1-4]

\section{Results and Discussion}

The electronic spectrum of $\{\mathbf{1}\} \mathrm{PF}_{6}$ (cf. Fig. 1) consists of two regions. An absorption band at $460 \mathrm{~nm}$ corresponds to a ligand-to-metal charge-transfer resonance that is responsible for the characteristic red-brown color of this crystalline compound. Irradiation into this band at cryogenic temperatures results in di-nitrogen cleavage and photo-oxidation to $\{\mathbf{3}\}$. Very intense absorption features for wavelengths shorter than $380 \mathrm{~nm}$ are likely to result from a number of different electronic excitations of yet unknown nature. Excitation at such short wavelengths results in photo-reduction to \{2red\} and release of azide radicals. 


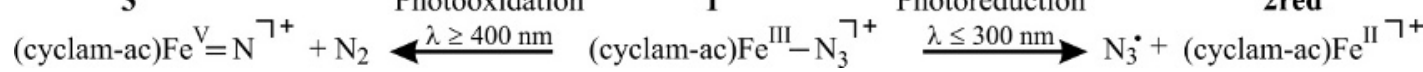

The infrared (IR) spectrum of $\{\mathbf{1}\} \mathrm{PF}_{6}$ (cf. Fig. 1) features two important bands namely, the acetato$\mathrm{CO}$ vibration of the cyclam-ac ligand at $1689 \mathrm{~cm}^{-1}$ and the stretching vibration of the $\mathrm{N}_{3}$ ligand at $2051 \mathrm{~cm}^{-1}$. In our studies, these two resonances serve as sensitive marker bands for a time-resolved IR detection of the photo-induced dynamics.
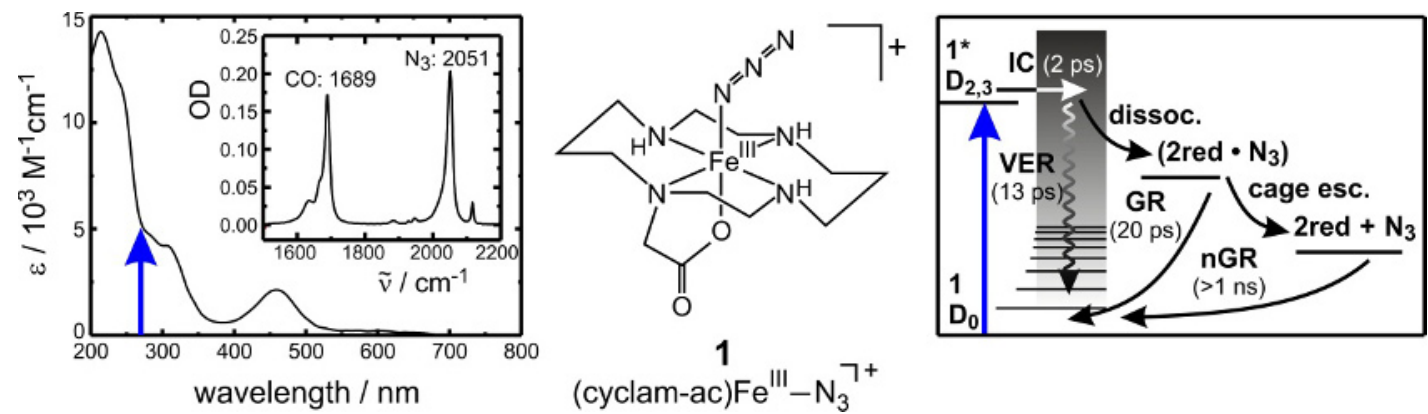

Fig. 1. Left: Electronic and IR (inset) spectra of complex $\{\mathbf{1}\}$ in liquid acetonitrile at $298 \mathrm{~K}$. Right: Hierarchy of dynamical processes governing the femtochemistry of $\{\mathbf{1}\}$ at $266 \mathrm{~nm}$.

The dynamic IR spectrum in the acetato- $\mathrm{CO}$ and $\mathrm{N}_{3}$ stretching region following excitation of $\{\mathbf{1}\} \mathrm{PF}_{6}$ in acetonitrile solution with 150-fs, 266-nm pulses is shown in Fig. 2a and b for a few representative pump-probe delays between $400 \mathrm{fs}$ and 80 ps. Complementary kinetic traces recorded on the two characteristic parent resonances are displayed in Fig. $2 \mathrm{c}$ and d. Immediately after excitation, the two marker bands of $\{\mathbf{1}\} \mathrm{PF}_{6}\left(1689 \mathrm{~cm}^{-1}\right.$ and $\left.2051 \mathrm{~cm}^{-1}\right)$ are bleached because the electronic ground state is depopulated by the pump pulse. These two spectral holes are partially refilled within $100 \mathrm{ps}$, but a residual bleach remains indicating that the ground electronic state is not fully replenished. From the ratio between residual bleach at 100 ps to initial bleach at zero delay, a primary photolysis quantum yield of $40 \%$ can be determined. This quantity corresponds to the fraction of electronically excited $\{1\} \mathrm{PF}_{6}$ that is able to decay to photochemical products, presumably to $\mathrm{N}_{3}$ radicals and complex $\{2$ red $\}$ (photo-reduction).
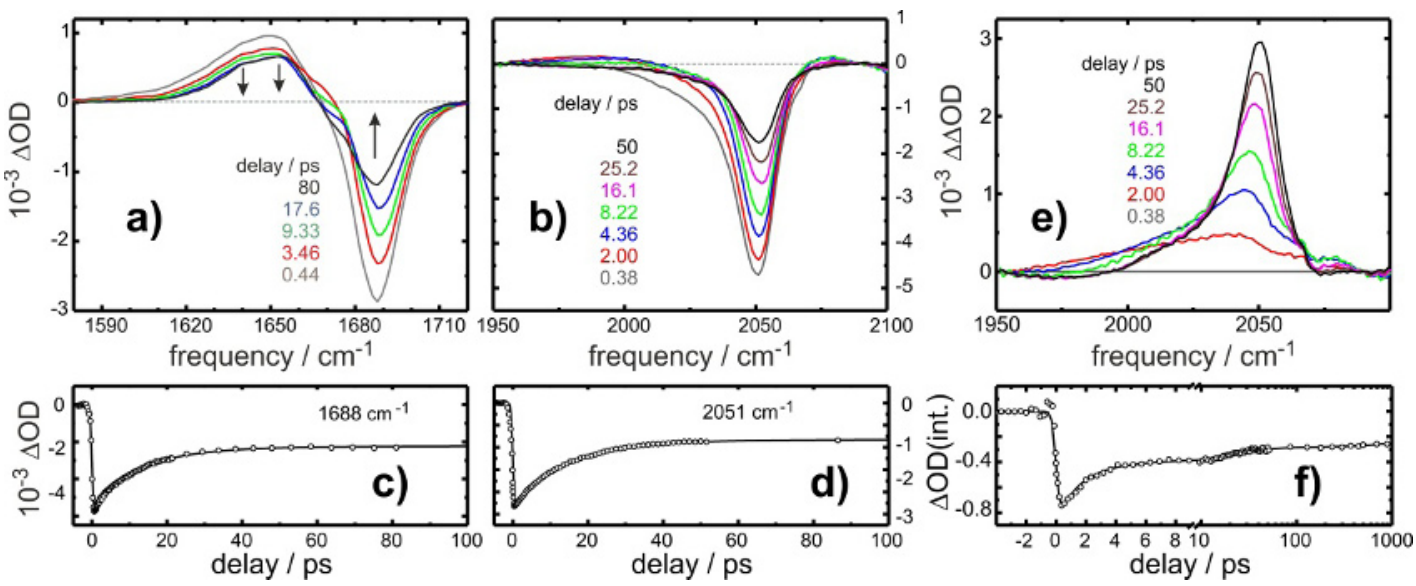

Fig. 2. Time-resolved UV pump/IR probe-spectra of $\{\mathbf{1}\} \mathrm{PF}_{6}$ in in the acetato-CO (a) and the azido stretching region (b). Kinetic traces of the bleaching peaks are shown in $\mathrm{c}$ and $\mathrm{d}$. The time-resolved azido stretching band of the replenished ground state is displayed in (e) and its band integral as a function of time-delay is shown in (f). Solid curves are multi-exponential fits. 
Fig. 2 reveals further that immediately after excitation (i.e. 400 fs delay), a broad absorption in the acetato-CO region appears at $1650 \mathrm{~cm}^{-1}$, which is shifted to lower frequencies relative to the parent absorption. One might be tempted to conclude that this feature is a spectral signature of the electronically excited Franck-Condon region of $\{\mathbf{1}\} \mathrm{PF}_{6}$. However, a careful line-shape analysis demonstrates that the azide radical also contributes to this induced absorption on picosecond time scales. Within the first 3 ps, the amplitude of this induced absorption decays by about $20 \%$. During the same time period, the amplitude of the ground state bleach at $1689 \mathrm{~cm}^{-1}$ also recovers by roughly $20 \%$. However, whereas the bleaching signal continues to decay by another $50 \%$ during the following $80 \mathrm{ps}$, the absorptive signal remains more or less constant. This can be interpreted in a consistent fashion only by assigning the initial fast decay within the first few picoseconds to the actual depopulation decay of the electronically excited state. This process might involve a ligand dissociation and/or the internal conversion back to the electronic ground state. Then, the question arises as to why the recovery of the ground state bleach is not completed within 3 ps. Indeed, the ground state bleaching signals at $1688 \mathrm{~cm}^{-1}$ and $2050 \mathrm{~cm}^{-1}$ recover bi-exponentially with time constants of $\sim 2$ ps and 13 ps (cf. Fig. $2 d$ and e).

Further information regarding the slow ground state dynamics was obtained from the $\mathrm{N}_{3}$ stretching band. Subtracting an early-time transient spectrum (e.g. at $400 \mathrm{fs}$ ) from the dynamic spectra at later times, yields exclusively the dynamically evolving spectrum of the "reformed" ground-state parent complex (i.e. complex $\{\mathbf{1}\}$ after recombination and/or internal conversion). At early delays, such difference spectra are highly asymmetrically broadened and are peaked at significantly lower frequencies as compared to the steady-state absorption of the azide ligand (cf. Fig. 2e). With increasing delay, the resonance narrows and its peak frequency approaches that of the equilibrated parent complex. This peculiar spectro-temporal evolution is highly characteristic for polyatomic molecules that reside in a non-thermal vibrational energy distribution and that transfer their excess energy onto the surrounding solvent particles (VER). Therefore, the 13 ps-process represents the VER dynamics of ground-state $\{\mathbf{1}\} \mathrm{PF}_{6}$.

Finally, to disentangle the pure electronic population dynamics associated with the recovery of the parent complex, $\{\mathbf{1}\} \mathrm{PF}_{6}$, from the VER dynamics within its electronic ground state, the timedependent band integral of the azide bleaching signal was calculated (cf. Fig. 2e, middle panel). This trace shows that the ground-state is re-populated in a bi-phasic fashion: (i) via internal conversion within 2 ps and through geminate recombination of the fragments following Fe-N bond breakage and azide radical formation within several tens of picoseconds. The hierarchy of primary events that follow the initial 266-nm excitation is schematically depicted in the right panel of Fig. 1. Further experiments of the photo-oxidation pathway are currently underway.

\section{Acknowledgements}

Financial support by the Deutsche Forschungsgemeinschaft through the Collaborative Research Center SFB 813 "Chemistry at Spin Centers" is gratefully acknowledged.

\section{References}

1. J. F. Berry, E. Bill, E. Bothe, S. DeBeer George, B. Mienert, F. Neese, K. Wieghardt, Science, 312, 1937 (2006)

2. C. A. Grapperhaus, B. Mienert, E. Bill, T. Weyhermüller, K. Wieghardt, Inorg. Chem. 39, 5306 (2000)

3. H. Vennekate, D. Schwarzer, J. Torres-Alacan, O. Krahe, A. C. Filippou, F. Neese, P. Vöhringer, Phys. Chem. Chem. Phys. 14, 6165 (2012)

4. J. Torres-Alacan, O. Krahe, A. C. Filippou, F. Neese, D. Schwarzer, and P. Vöhringer, Chem. Eur. J. 18, 3043 (2012) 